\section{New database could lead to antibiotic overuse}

Pharmaceutical companies often collect data from physicians in order to guide marketing efforts, but a new database sponsored by Bayer will be the first to use information on the incidence of respiratory tract infections (RTI) to help market antibiotics directly to consumers.

Updated weekly, the RTIalert will provide detailed geographical information about the local incidence of bacterial and viral infections, allowing patients, physicians and industries to respond to outbreaks rapidly during the winter cold season. However, some have expressed concern that the system-which is closely linked to the advertising campaign for Bayer's antibiotic Avelox (moxifloxacin)could exacerbate the problems of antibiotic overprescription and subsequent bacterial resistance.

RTIalert tracks diagnostic information from 30,000 physicians across the US in order to generate the 'RTI Index,' a number between one and ten indicating the relative incidence of bacterial respiratory tract infections in a given town or city.

Rather than recruiting physicians di- rectly, Surveillance Data Incorporated (SDI), the company that designed the system for Bayer, established contracts with companies that handle medical billing. For billing purposes, doctors assign a diagnostic code whenever a patient is treated and these codes, stripped of identifiable patient information, are forwarded to SDI. The index for a patient's town or city is available through the Internet and a toll-free number.

Andrew Cress, president of SDI, explains that collecting codes from billing companies is "a terrific way to gather data from a disease surveillance perspective because you get enormous sample sizes. W e're seeing five to seven percent of all outpatient visits in a given week in the US," or 85-100 million visits per year. Because the company does not interact directly with the doctors, there is also less chance of introducing a bias when the data are collected.

SDI has developed similar databases for other pharmaceutical companies to track influenza, allergies and other conditions, but the focus of the RTIalert on bacterial infections raise a unique concern: "It may increase pressure on doctors to prescribe antibiotics-unnecessarily in many cases," says Howard Bauchner, a pediatrician at the Boston University School of Medicine who has done research on the problem of antibiotic overprescription.

While conceding that some patients may use the RTI Index to try to pressure doctors for antibiotics, Cress responds that, "I think at the end of the day it comes down to that physician managing that interaction," and adds that recent educational programs have helped to reduce overprescription. Still, says Bauchner, "in general, when docs are pressured they often do prescribe, so this may make it more difficult to curb the use of oral antibiotics."

Paul Iannini, clinical professor of medicine at Yale University and scientific advisor to the RTIalert, asserts that the system will actually help combat the problem of overprescription: "if you want to know if the current outbreak is being coded by your peers as more likely viral than bacterial, [the RTIalert] will tell you," encouraging physicians to avoid using antibiotics when the RTI Index is low.

Alan Dove, Philadelphia

\title{
New bioterrorism institute enters burgeoning field
}

Almost \$2 million has been set-aside in budget legislation to establish a new bioterrorism research program at the University of Pittsburgh and the Carnegie Mellon Research Institute in Pittsburgh.

The Biomedical Security Institute (BMSI) will focus initially on developing computing tools to identify and combat disease outbreaks and acts of bioterrorism, an issue that has gained considerable public and political attention in recent years. Indeed, some have expressed concern that the political popularity of bioterrorism prevention is leading to a proliferation of research programs that lack coordination.

In this year's budget for the Centers for Disease Control and Prevention (CDC), \$177 million-50\% more than the National Science Foundation spent on all types of health related RTD in 1999-has been set aside to address bioterrorism, and $\$ 1$ million of this was earmarked by Arlen Specter (R., PA) to establish the BMSI. An additional $\$ 1$ million for the Institute will come from Congressionally mandated bioterrorism prevention funding through the Agency for Healthcare Research and Quality.

According to government data, the $\$ 177$ million CDC budget for bioterrorism is $50 \%$ more than the National Science Foundation spent on all types of health-related research and development in 1999.

BMSI will improve databases and "data mining" software that speed the detection of suspicious disease outbreaks by coordinating diagnostic information from hospital emergency rooms and primary care physicians. Other work will range from developing biosensors to training federal, state and local officials in responding to a biological attack.

Samuel Watson, co-director of the BMSI, says, "our focus is improving public health of Americans and countering bioterrorism and we think you do both of them simultaneously by improving infectious disease efforts, by improving public health measures, and by improving surveillance."

Watson hopes that BMSI will also be able to address the proliferation of biodefense research programs: "I have proposed to CDC that they let us try and get all the bioterrorism centers around the country together and make some sense of what they're doing."

But not everyone is convinced that the money is being spent wisely. "I know nothing whatsoever about the Pittsburgh initiative nor am I aware of any special competence that they might have that permits them to undertake special activities," says Donald Henderson, director of the Center for Civilian Biodefense Studies at Johns Hopkins University. He adds that currently "there are several other enigmatic dollops of funding provided to other institutions that hitherto didn't seem to have much interest in [bioterrorism]."

\section{Alan Dove, Philadelphia}

